\title{
GLOBALIZACIÓN, INTERDEPENDENCIA
}

\author{
COMPLEJA Y MUNDIALIZACIÓN：LA DIALÉCTICA \\ ENTRE LO GLOBAL Y LO LOCAL*
}

Ricardo Sebastián Piana ${ }^{(a)}$, Juan Cruz Tisera ${ }^{(b)}$

GLOBALIZATION, COMPLEX INTERDEPENDENCE AND GLOBALIZATION:

THE DIALECTIC BETWEEN THE GLOBAL AND THE LOCAL

GLOBALIZAÇÃO E GLOBALIZAÇÃO INTERDEPENDÊNCIA COMPLEXA:

A DIALÉTICA ENTRE O GLOBAL E O LOCAL

Fecha de recepción: 10 de enero del 2017

Fecha de aprobación: 2 de agosto del 2017

Sugerencia de citación:

Piana, R.S. y Cruz Tisera, J. (2017). Globalización, interdependencia compleja y mundialización: la dialéctica entre lo global y lo local. Razón Crítica, 3, 145-173, doi: http://dx.doi.

org/10.21789/25007807.1240

* El presente artículo se estructura como resultado parcial y a partir de la asistencia de investigación desarrollada para el proyecto "Global: la influencia de las políticas y buenas prácticas globales sobre las políticas públicas en la provincia de Buenos Aires”, dirigido por Ricardo S. Piana y Rubén D. Guerra, Facultad de Ciencias Jurídicas y Sociales, Universidad Nacional de La Plata (UNLP). Instituto de Integración Latinoamericana. Acreditación de Proyectos Bienales, Investigación y Desarrollo (Proyectos I+D) 2016. Secretaría de Ciencia y Técnica, UNLP. (a) Abogado y Doctor en Ciencias Jurídicas por la Universidad Nacional de La Plata, y Doctor en Ciencia Política por la Universidad del Salvador. Es adjunto ordinario de derecho político en la carrera de abogacía de la UNLP, y titular de Derecho y teoría constitucional en la USAL, Argentina. http://orcid.org/0000-0001-8743-8942.r_piana@yahoo.es

(b) Doctor en Relaciones Internacionales, Universidad del Salvador. Docente titular Seminario de Doctorado en Metodología de las Ciencias Sociales, del Doctorado en Relaciones Internacionales y del Doctorado en Ciencia Política de la Universidad del Salvador, Argentina. http://orcid. org/0000-0001-5122-2728. jctisera@gmail.com 


\section{R E S U M E N}

Desde el ámbito de las Relaciones Internacionales, pero con un abordaje y una perspectiva compleja y multidisciplinar, el artículo busca identificar características del proceso de influencia de doble vinculación entre la sociedad internacional y los gobiernos subnacionales (provinciales y municipales), describiendo un fenómeno que ha sido identificado como glocal. El resultado señala la conformación de una nueva sociedad mundial y global, que requiere de una reconstrucción teóricometodológica para comprender fenómenos distintos (y no descriptos) al momento de consolidar las relaciones internacionales como objeto autónomo de estudio. Se analizan los tres procesos señalados y sus relaciones, centrándose especialmente en la dialéctica global-local, desde una perspectiva que ha sido identificada como una nueva sociedad internacional, ahora, global. Procedemos a la adopción de un modelo de análisis determinado a través del concepto política internacional subnacional que se ejerce en el ámbito local.

PALABRAS CLAVE: globalización, glocalización, interdependencia compleja, mundialización. 


\section{A B S T R A C T}

Elaborated from the field of International Relations, but with a complex and multidisciplinary approach and perspective, the article seeks to identify characteristics of the process of double-linking influence between international society and subnational (provincial and municipal) governments, describing a phenomenon that has been denominated as glocal.

The result is that there is a new global society, which requires a theoretical and methodological reconstruction to understand different (and not described) phenomena that arose when International Relations were established as an autonomous object of study. The three processes described are analyzed and so are their relationships. The global-local dialectic is emphasized from a perspective that has been identified as a new international society, now, global. We adopt then a model of analysis based on the concept of International Subnational Policy that is exercised at the local level.

KEY WORDS: globalization, complex interdependence.

\section{R E S U M O}

A partir do âmbito das Relações Internacionais, mas com uma abordagem e uma perspectiva completa e multidisciplinar, o artigo busca identificar características do processo e sua influência de dupla vinculação entre a sociedade internacional e os governos subnacionais (províncias e municípios), descrevendo um fenômeno que tem sido identificado como glocal. O resultado assinala a conformação de uma nova sociedade mundial e global, que necessita uma reconstrução teórico-metodológica para compreender fenômenos distintos (e não descritos) no momento de consolidar as Relações Internacionais como objeto autônomo de estudo. São analisados os três processos assinalados e suas relações, concentrando especialmente na dialética global-local, até uma perspectiva que tem sido identificada como uma nova sociedade internacional, agora, global. Procedemos a adoção de um modelo de análise determinado através do conceito Política Internacional Subnacional que se exerce no âmbito local.

PALAVRAS-CHAVE: globalização, glocalização, interdependência complexa. 


\section{N T R O D U C C I Ó N}

El presente artículo pretende desarrollar y repensar algunos conceptos clave de las relaciones internacionales que giran en torno al concepto de globalización desde una perspectiva compleja y multidisciplinar. En la actualidad nos encontramos en una sociedad internacional de carácter global, o como los académicos sostienen, una era postwestfaliana, muy diferente a la existente hasta fines del siglo pasado, dado el debilitamiento de algunos de los principales elementos que se afirmaron a partir de 1648 y marcaron claramente los caracteres de la sociedad internacional hasta la segunda mitad del siglo XX (Buzan y Little, 2000, pp. 2-6).

¿Qué es la globalización?; ¿es un cambio de escala, una continuidad o se trata de un fenómeno totalmente nuevo que envuelve a todo el globo terráqueo? El término globalización se ha trasfigurado en una suerte de cliché (Held, Mcgrew, Goldblatt y Perraton, 2003, p. 13). El propio Ulrich Beck (2004, p. 40) señaló que el concepto globalización es la palabra o eslogan peor empleado, peor definido, pero, a su vez, la palabra políticamente más eficaz de los últimos años. Siguiendo este pensamiento, Zygmunt Bauman (2010, p. 7) señaló claramente que el fenómeno globalización está en boca de todos, transformándose rápidamente en la palabra de moda, hasta un fetiche, la llave que abriría todos los misterios.

Dado que las principales corrientes teóricas dan por sentado que los problemas y las oportunidades de la globalización, sobre todo a partir de las respuestas políticas dadas, se encuentran en el nivel macro, pretendemos cuestionar ese supuesto y explorar una 
reciente línea teórica que ha llamado la atención sobre la escala con que hay que observar el fenómeno.

Centrando el desarrollo de nuestro marco teórico en relación con el lema "pensar globalmente y actuar localmente" (Cruz y Bodnar, 2008, pp. 13-25), buscamos identificar características del proceso de influencia de doble vinculación entre la sociedad internacional y los gobiernos subnacionales (provinciales y municipales), describiendo un fenómeno que ha sido identificado como glocal. La política internacional subnacional ${ }^{1}$ es un fenómeno relativamente novedoso, y que se encuentra en constante redefinición, un proceso que ha comenzado a generar conceptos e interpretaciones, a veces contradictorias, que requiere un estudio que lo distinga de otras orientaciones con las que se lo suele confundir (Calvento, 2015). Para no caer en este error, creemos necesario profundizar el debate teórico y ampliar los estudios empíricos, lo que genera nuevos interrogantes que permiten comprender el proceso actual.

Abordaremos a la globalización como un fenómeno contemporáneo y determinado a partir de su multidimensionalidad, distinguiendo los desafíos que se plantean para los estados, en particular, cómo este contexto repercute en la formulación de políticas públicas locales, pero cómo, a su vez, estas retroalimentan esos paradigmas y buenas prácticas.

En la primera parte del artículo, nos detenemos en un aspecto previo, que no siempre es tratado en los artículos de investigación, la función de la teoría y el método en el campo disciplinar de las relaciones internacionales, pero que se nos presenta como fundamental para trabajar el tema objeto de este estudio. En definitiva, pretendemos responder a los siguientes cuestionamientos: ¿cuáles son los enfoques teóricos y los modelos de las relaciones internacionales?, y ¿cómo generar la construcción de un enfoque integral sobre la realidad de los actores subnacionales en el contexto actual?

1 Tomamos la definición de la política internacional subnacional como "la decisión política y la herramienta pública de los gobiernos locales que se ocupa de impulsar la inserción internacional, a través de una estrategia y objetivos tendientes a aprovechar, articuladamente, las oportunidades del contexto exterior con las necesidades del territorio" (Calvento, 2016, p. 309). 
En el segundo apartado, comenzamos una aproximación conceptual al fenómeno de la globalización: no solo una delimitación temática, sino también identificamos un campo de discusión. Sin embargo, como veremos en el siguiente apartado, la globalización supone, (pero es distinta) a la mundialización: esta se desarrolla y se conceptualiza antes que el proceso globalizador y, sin embargo, no ha sido absorbido por esta. Producto de la expansión y conquista europea, se modificó la percepción del mundo, el tiempo y se crearon los presupuestos para la existencia de una sociedad internacional global.

Seguidamente, nos proponemos estudiar la creciente interdependencia como una de las dinámicas de la actual sociedad internacional global. La interdependencia se desprende de la conceptualización de la globalización y comienza a adquirir características autónomas, hecho relevante para comprender las profundas transformaciones que experimenta la actual realidad internacional.

Finalmente, desarrollamos el concepto de lo glocal como una nueva herramienta conceptual para comprender la interdependencia compleja de lo global y lo local. Es en este contexto que los actores subnacionales buscan generar políticas públicas que permiten potenciar las acciones en el exterior.

\section{El valor de la teoría: una mirada desde las relaciones internacionales}

Buscamos en este apartado profundizar los aspectos teóricos y metodológicos de las relaciones internacionales, conceptualizando la inserción internacional de los actores subnacionales, pretendiendo generar modelos explicativos que nos permitan entender la inserción internacional de actores no estatales sobre la articulación dialéctica global-local.

Desde el campo de las relaciones internacionales, proponemos establecer un marco teórico que analiza el despertar internacional de los actores subnacionales. En un contexto denominado sociedad internacional global, se determinan las modificaciones del carácter central y unitario del Estado-nación propuesto por el realismo político. Keohane y Nye (1988) señalan el surgimiento 
de nuevos actores, gubernamentales y no gubernamentales, que conforman una nueva trama de relaciones transnacionales e intergubernamentales.

Las relaciones internacionales no están ajenas a las diferentes controversias en torno a sus cuestiones teóricas; al igual que las otras disciplinas de las ciencias sociales se encuentran en una búsqueda continua de teorías que permitan el abordaje de los cambiantes fenómenos que estudian. Hoffmann señaló que "la teoría constituye el principio de orden en una disciplina" (1963, p. 26). El problema, más allá de esta definición, es que la teoría no posee un sentido unívoco y es por ello que necesitamos determinar los diversos contenidos y aspectos de su formulación.

Karl Deutsch (1966) sostuvo que una teoría nace a partir de una conjetura y solo será viable en la medida que se confirme. Pero en la actualidad no existe una única teoría de las relaciones internacionales que nos permita dar explicaciones satisfactorias y menos aún predecir efectos. Ángel Tello sostiene que

[...] la teoría no es algo pétreo, sino un cuerpo de hipótesis válido hasta que ocurre un accidente denominado refutación. Por ello corresponde considerar en este caso el modelo de análisis hipotético deductivo basado en premisas e hipótesis que faciliten una explicación de los fenómenos mundiales (Tello, 2013, p. 560).

Siguiendo los aportes de Kenneth Waltz podemos señalar que el término teoría se ha utilizado en las relaciones internacionales para trabajos meramente descriptivos. Sin embargo, esta afirmación no descalifica a los aportes de las relaciones internacionales, pues el mismo autor reconoce que hay dos definiciones de teoría, ambas válidas: una primera señala que “[...] las teorías son conjuntos de leyes que pertenecen a una conducta o fenómenos particulares" (1979, p. 10), y, en este sentido, una teoría es una representación simplificada de una parte de la realidad, es decir, organiza fenómenos, vincula hechos y demuestra cómo los cambios en los fenómenos de la realidad conducen a otros cambios. El segundo significado del término sostiene que "las teorías explican las leyes" (Waltz, 1979, p. 16). De esta segunda definición surgen, como veremos, varios problemas y cuestionamientos, ya que dicha 
definición resulta más aplicable a las ciencias naturales que a las ciencias sociales.

Duroselle cree que es injusto hacer una analogía entre las ciencias duras y las sociales, así como también lo es la pretensión de identificar a las ciencias solo como conocimiento científico riguroso y agrega que "como todas las ciencias humanas, la teoría de las relaciones internacionales no pueden actualmente, ni podrá por mucho tiempo, rebasar la fase empírica” (1998, p. 16). Especialmente en las relaciones internacionales, en la coyuntura de la investigación actual, lo que se produce y publica es puramente descriptivo ${ }^{2}$, y si bien la finalidad será la misma, la verdad objetiva, no podremos tratar del mismo modo los dominios de la mente humana: las "leyes" en las ciencias sociales son leyes a medias, leyes incompletas o causalidades fuertes, pero no necesarias. Las ciencias humanas no pueden fundamentar sus supuestos siguiendo los modelos de las ciencias exactas, sino que deben construir sus métodos propios ${ }^{3}$.

La teoría de las relaciones internacionales necesita de modelos que permitan reflejar la realidad internacional. Un modelo nos permite dar una descripción simplificada de la realidad y, por otro lado, representar una teoría. Es importante destacar que si este se aleja de la realidad, se torna insuficiente y no cumple su función.

Como señala Calduch Cervera,

[...] por modelo entendemos una abstracción de la realidad que nos ayuda a comprenderla mejor. Puede ser implícito o explícito, consciente o inconsciente, literario o científico, realista o idealista, más influido por el pasado o más influido por el futuro o el presente, experimental o racional. Todo modelo es una simplificación de la realidad en la que resaltan unos elementos y se ignoran o difuminan otros. Simplificar no es necesariamente malo. Al contrario, todo ejercicio intelectual requiere cierto grado de simplificación y de síntesis para facilitar

\footnotetext{
2 Según Duroselle (1998), los fenómenos que estudiamos en las relaciones internacionales son acontecimientos, es decir, todos ellos se distinguen por ser singulares, carentes de identidades absolutas. Ello hace que estemos, según este autor, ante un conocimiento puramente empírico.

3 Obviamente que los métodos propios para construir una ciencia de las relaciones internacionales serán muy diversos, y hay grandes pensadores que han pretendido tomarlos de otra ciencia humana: los aportados por la historia, como propone Duroselle, la economía, como propone por su parte, Raymond Aron.
} 
la comprensión. Es, posiblemente, la única forma de comprender el mundo que nos rodea (2000, p. 32).

Por su parte, Marcel Merle formula un concepto más restringido:

El término modelo debe tomarse aquí en un sentido moral.

Designa un esquema sintético y abstracto que ordena los elementos de la realidad en una construcción rigurosa. La construcción de un modelo parte de una serie de observaciones concretas o experimentales sobre hechos o datos previamente cuantificados (1986, p. 138).

Debemos destacar que la finalidad no deberá ser la de una mera reproducción de la realidad, sino la de representar una configuración que nos permita la comprensión y la determinación de los comportamientos que pretendemos investigar.

La modelización teórica en nuestra disciplina se ha visto enriquecida por sus instrumentos metodológicos y el desarrollo de sus diferentes debates que han abierto un camino que estamos empezando a recorrer. ¿Cómo construimos teoría? La primer repuesta a este interrogante es que debe ser creativamente: en algún punto surge una idea brillante y novedosa que se aleje de lo común. Además " $[\mathrm{u}]$ na teoría no consiste en los acontecimientos vistos y las asociaciones registradas, sino que es la explicación de ellos" (Waltz, 1979, p. 21). En definitiva, una teoría nos debe permitir el ordenamiento de fenómenos, conectando hechos que, de otro modo, serían inconexos y los cambios a otros fenómenos.

Como ya hemos señalado, una teoría genera una simplificación de la realidad: es una guía de interpretación para poder entender qué ha sucedido, qué es lo que sucede y qué es lo que sucederá: "la construcción de una teoría es una tarea primaria. Es necesario decidir en qué cosas concentrarse con el objeto de tener una buena posibilidad de idear explicaciones de las estructuras y acontecimientos internacionales que nos interesan" (Waltz, 1979, p. 31). Cuando el investigador realiza una simplificación, organiza la explicación e interpreta la realidad, es decir, realiza una teoría y toma diferentes caminos debido a que no siempre se realiza un mismo procedimiento de simplificación, organización e interpretación. 
Pero el camino de la simplificación no debe conducirnos al monismo o a la pretensión de explicar todo a partir de una única causa, porque esto no implica una simplificación, sino un reduccionismo. El reduccionismo no explica, sino que oculta variables. Por el contrario, la simplificación es un criterio metodológico y filosófico de antigua data según el cual en igualdad de condiciones, la explicación más sencilla suele ser la más probable ${ }^{4}$. Como ha dicho Waltz, "[...] en la época actual, estamos obligados a teorizar sobre una humanidad de la cual cada miembro obedece alternativa y simultáneamente a motivaciones heterogéneas. En la actualidad, imponer una explicación monista es lanzarse en el callejón sin salida de las ciencias muertas” (1979, p. 337).

Estas aclaraciones metodológicas previas y del campo disciplinar son necesarias para abordar la complejidad del tema que estudiamos. Como veremos, nos encontramos con un fenómeno complejo que asume todos los elementos de una ideología que, como tal, se presenta como inmodificable. Si asumimos esa hipótesis, la globalización, que es solo una teorización-generalización de fenómenos individuales, asume una impronta inabarcable, imposible de modificar.

El modelo teórico-metodológico propuesto pretende consolidar la tesis que afirma que lo local rompe con la lógica internacionalista clásica estatocéntrica, lo que genera múltiples conexiones y sujetos. La evidencia teórica se sustenta a través de la política internacional subnacional $^{5}$, que se ejerce en el ámbito local: observaremos en un escenario de estrategia de desarrollo local, donde el accionar internacional "[...] debe constituir un componente esencial si dicha estrategia pretende ser integral y enfrentar los múltiples desafíos que presenta un sistema internacional complejo, interdependiente y cambiante" (Costamagna y Foglia, 2011, p. 8).

4 Es conocido como principio de parsimonia conforme lo formulara el fraile franciscano inglés Guillermo de Ockham.

$5 \quad$ El término política internacional subnacional se utilizó por primera vez en la Argentina en la obra de Luis Maira (2010). 


\section{Planteamientos conceptuales sobre la globalización}

Las últimas décadas del siglo XX han traído consigo una dinámica de cambios profundos a partir del desarrollo de la globalización, lo que ha ocasionado una profunda transformación en la sociedad internacional. Como sostuvo Truyol, estamos frente a una "[...] verdadera mutación de la sociedad internacional” (1993, p. 26).

Como analizaremos más adelante, no es este un fenómeno nuevo, sino que la sociedad internacional en la que vivimos se ha configurado progresivamente desde la paz de Westfalia (1648) hasta su actual modelo alcanzado hacia el final de la Segunda Guerra Mundial ${ }^{6}$.

Es indudable que, como todas las síntesis y todos los paradigmas, esta definición comporta simplificaciones excesivas y reduccionismos, pero sería inútil negarse a utilizar una terminología que sirve, al menos, para delimitar una temática, para identificar un campo de discusión, aunque sea de forma aproximativa.

De forma liminar, podemos decir que la globalización connota la ampliación e intensificación de relaciones sociales, económicas y políticas a través de regiones y continentes. Es un fenómeno multidimensional que abarca muchos procesos diferentes y opera en múltiples escalas temporales (Held, et al., 2002).

La globalización está lejos de ser un fenómeno singular, y, pese a ello y a que trasluce un giro general en la organización de la actividad humana y un desplazamiento del poder hacia pautas transcontinentales o intrarregionales, puede adoptar formas distintas y seguir trayectorias diversas a través del espacio económico, político o de otra índole. Puede también

6 Ciertamente, puede objetarse que las interrelaciones globales no son algo nuevo, pues su importancia política ha sido destacada y patente desde hace largo tiempo. En efecto, muchos autores críticos destacan la densa red de interrelaciones globales que comenzaron a emerger con la primera expansión de la economía mundial y con la configuración del Estado moderno a fines del siglo XVI. Sin embargo, la novedad del sistema global actual radica en la intensificación crónica de los modelos de interdependencia, mediatizados por fenómenos tales como la industria de las comunicaciones moderna y la nueva tecnología de la información; la propagación de la globalización dentro y a través de nuevas dimensiones de interrelación: tecnológica, organizativa, administrativa y jurídica, entre otras, cada una con una lógica interna y su propia dinámica de cambio. 
crear tendencias conflictivas así como complementarias en la determinación de las relaciones de poder y autoridad.

El término globalización no solo es polisémico, vago y ambiguo, sino que es usado según las conveniencias para denostar, alabar o simplemente describir fenómenos propios de la realidad internacional:

Estamos, en este sentido, ante un término comodín, fácil de usar, que por eso mismo, en muchos casos, no sabemos exactamente cuál es su significado y alcance y que, consecuentemente, con frecuencia más que aclararnos la realidad social que tratamos de explicar lo que hace es confundirnos sobre la misma (Arenal, 2009, p. 128).

Por lo tanto, trataremos de aclarar el significado desde una perspectiva disciplinar específica, la de las relaciones internacionales, sin perjuicio de reconocer el desarrollo interdisciplinar del concepto, que nace con conformación de una sociedad internacional global ${ }^{7}$.

7 Creemos necesario hablar de una sociedad internacional global reconociendo la existencia de otros términos, como escenario internacional; sistema internacional; vida internacional, entre otros tantos compartidos por la academia. En nuestra opinión, el término adecuado es el de sociedad internacional; por ello, hacemos referencia a la existencia de tres realidades sociales que coexisten e interactúan. Según Arenal, "Estas tres realidades son la sociedad o el sistema de comunidades políticas o estados o, con otras palabras, el sistema político-diplomático, el sistema transnacional y la sociedad humana" (Arenal, 2005, pp. 453-464). Bull hace una distinción entre sistema internacional y sociedad internacional; al primero lo define como dos o más estados que tienen contacto suficiente entre ellos, mientras que la sociedad internacional existe cuando un grupo de estados, conscientes de determinados intereses y valores comunes, forman una sociedad en el sentido de que se conciben a sí mismos como sujetos a un conjunto de normas en su relación con los otros, y participan en el funcionamiento de las instituciones comunes". Como señalamos anteriormente, la sociedad internacional presupone al sistema internacional, y esta está históricamente fundada sobre una cultura o civilización común. Según los planteamiento del autor, la sociedad internacional tiene cuatro objetivos universales de vida: la preservación del sistema y la sociedad en sí mismos; el mantenimiento y la independencia de la soberanía de los estados (sobre todo la externa); el mantenimiento de la paz como ausencia de guerra entre los estados (este objetivo se diferencia de Bobbio quien sitúa a la paz en primer término), y, por último, la estabilidad de las estructuras que posibilitan cumplir la palabra y los contratos, limitando de este modo la violencia (Bull, 1977). Otros teóricos identifican dos realidades haciendo referencia al sistema transnacional y la sociedad humana. Rosenau (1990), Hoffmann (1990, pp. 115-122) y Nye (2003, pp. 66-67) sostienen que lo que históricamente ha variado de una sociedad internacional a otra ha sido la importancia y el protagonismo que cada una de esas realidades sociales han tenido en las sociedades internacionales del momento y las interacciones que se han establecido entre ellas. "Con todo, de las tres realidades sociales que conforman la sociedad internacional es, lógicamente, el sistema político-diplomático, en cuanto expresión del fenómeno de descentralización del poder, el que proporciona al menos formalmente los rasgos definitorios de cada sociedad 
Hay tantas definiciones como autores estudian el fenómeno de la globalización. Los académicos Held et al. sostienen que la globalización:

[...] es un proceso (o una serie de procesos) que engloba una transformación en la organización espacial de las relaciones y las transacciones sociales, evaluada en función de su alcance, intensidad, velocidad y repercusión, y que genera flujos y redes transcontinentales e interregionales de actividad, interacción y del ejercicio del poder (2002, p. 49).

El propio Held se arriesga a dar una definición que busca evitar malos entendidos sosteniendo que "la globalización connota la ampliación e intensificación de relaciones sociales, económicas y políticas a través de regiones y continentes. Es un fenómeno multidimensional que abarca a muchos procesos diferentes y opera en múltiples escalas temporales" (Held, 2014, p. 77)8.

Por su parte, Giddens define a la globalización como producto de su teoría de la modernidad, entendiendo que esta supone "[...] la intensificación de las relaciones sociales en todo el mundo, por las que se enlazan lugares lejanos de tal manera que los acontecimientos locales están configurados por acontecimientos que ocurren a muchos kilómetros de distancia” (1993, pp. 67-68). Esta definición supone el incremento de las relaciones mundiales y, por lo tanto, no se pueden concebir las políticas locales sin tener en cuenta una consecuencia lógica de la modernidad, sus repercusiones globales y cómo este escenario influye en la formulación de las mismas.

Brzezinski afirma que "el linaje intelectual de la globalización no se puede rastrear [...] en los clásicos [...] ni en una única fuente dogmática" (2007, p. 33). Antes bien, se trata de un concepto que se

internacional y el que genera normalmente la existencia de normas e instituciones comunes que regulan el funcionamiento de esa sociedad internacional, especialmente a nivel del propio sistema político-diplomático. Todo ello supone que de las tres realidades sociales que configuran la sociedad internacional es el sistema político-diplomático la única que tiene un relativo grado de institucionalización y formalización, que varía notablemente, como es lógico, en función de los distintos tipos de sociedad internacional que han existido históricamente" (Arenal, 2005, pp. 460-464).

8 "El fenómeno envuelve la ampliación y profundización de instituciones y relaciones económicas, sociales y culturales, en espacio y tiempo. De tal manera, las actividades cotidianas se ven crecientemente influenciadas por situaciones que ocurren en otros lugares del planeta, a la vez que las prácticas y decisiones de grupos o comunidades a nivel local pueden tener amplias repercusiones más allá de las fronteras” (Held, 1997, p. 14). 
fue popularizando por su uso en los medios, en la propaganda y en el mundo de los negocios, además de en libros de opinión, primero, y en trabajos académicos, después. De tal manera, al tiempo que la globalización se popularizaba, simultáneamente, se desarrollaba intelectualmente para ir convirtiéndose en casi una doctrina (Silva, 2009, p. 3-25). Desde esta mirada hay que distinguir un discurso ético sobre el fenómeno globalizador y, por lo tanto, deberíamos comprender a la globalización como un fenómeno explicado a partir de una ideología.

Es indispensable rechazar el supuesto según el cual la globalización es únicamente un fenómeno "objetivo" de tipo económico, cuando en realidad ese fenómeno es también y, podemos añadir, sobre todo, una ideología ${ }^{9}$. De la ideología posee todas las características: la sistematicidad que excluye cualquier desviación, el rechazo de la crítica, la pretensión de objetividad, la aspiración a expandirse, la consideración de las ideologías competitivas como superadas, el triunfalismo y la dureza ${ }^{10}$. La ideología de la globalización comporta, como todas las ideologías, una pérdida de bipolaridad grávida de consecuencias bajo el aspecto ético: quien se oponga/acepte será bueno/malo.

Se puede considerar a la globalización también como un proceso, sobre todo, referenciado a un conjunto de sucesos que se generan a partir de interacciones transnacionales, esto es, a partir de "[...] la creciente gravitación de los procesos financieros, económicos, ambientales, políticos, sociales y culturales de [carácter] mundial en [aquellos] de carácter nacional o regional” (Cepal, 2002, p. 17).

Roland Robertson (1992) y Ulrich Beck (2008) también han trabajado la cuestión conceptual de la globalización, pero añaden el factor local. Robertson es el autor que hace referencia por primera vez a la idea de la glocalización, permitiéndonos

9 Alan Touraine en el mismo sentido dice en "La globalización como ideología", publicado en el diario El País, el 29 de septiembre de 1996 que "[...] hoy estamos dominados por una ideología neoliberal cuyo principio central es afirmar que la liberación de la economía y la supresión de las formas caducas y degradadas de intervención estatal son suficientes para garantizar nuestro desarrollo [...] Esta ideología ha inventado un concepto: el de la globalización. Se trata de una construcción ideológica y no de la descripción de un nuevo entorno económico".

10 Por ejemplo, entre los ideólogos de la globalización, ha surgido el amor por una cierta TINA (There Is No Alternative). 
comprender "[...] la dialéctica existente entre lo global y lo local, entre los universalismos y los particularismos" (1992, p. 45). Beck define a la globalización como "[...] los procesos en virtud de los cuales los estados nacionales soberanos se entremezclan e imbrican mediante actores transnacionales y sus respectivas probabilidades de poder, orientaciones, identidades y entramados varios” (2008, p. 29). De esta manera, la globalización significa aproximación y encuentro, donde las políticas públicas locales encuentran un nuevo marco en relación a una nueva realidad internacional. Las políticas locales encuentran un escenario de oportunidades en relación a la articulación de interés, pero también implican exigencias.

Luego de esta enumeración de definiciones, nos proponemos entender su conceptualización a partir de los vínculos que juega con otros conceptos e ideas, como la de la creciente interdependencia, la sociedad internacional global, la mundialización, la glocalización, entre otros. Siguiendo a Ribas Mateos (2002) podemos distinguir tres criterios diferentes:

- El primero de ellos se establece en relación con el juego del pasado, una conceptualización del mundo desde una perspectiva unitaria. Este análisis lo identificaremos desde el proceso de mundialización.

- Un segundo criterio se encuentra determinado a partir de la condición espacio-temporal. Este proceso se vislumbra a partir de las interacciones entre los actores de la sociedad internacional, "derivado de la instantaneidad en las mismas y de la simultaneidad de los acontecimientos" (Arenal, 2009, p. 216). Este criterio será trabajado desde el tablero de la creciente interdependencia.

- El último criterio parte de las definiciones que implican el aumento de la conciencia, la percepción y la transnacionalización por parte de la sociedad internacional: los seres humanos toman conciencia de pertenecer a un mundo que se considera único. En nuestra opinión consideramos que este criterio es el correcto para el análisis de la dicotomía global-local.

Este escenario, al que denominamos sociedad internacional global, nos permitirá comprobar que lo universal (pensar globalmente) se 
establece como consecuencia a intervenir en lo particular (actuar localmente). Sin embargo, como veremos, este proceso global no puede pensarse sin la mundialización que nace con (o que hace nacer) la Edad Moderna.

\section{Mundialización}

¿Por qué hablar de mundialización?, ¿cuáles son sus características? ¿Implica un fenómeno distinto al de la globalización?

Antes de comenzar su conceptualización, debemos considerar que este proceso, que se inició en Europa con la Edad Moderna, fue posible merced a diversos factores con sus consecuentes efectos, desde la perspectiva de una sociedad internacional ${ }^{11}$ : en primer lugar, debemos referenciar el surgimiento de los estados soberanos y el avance del desarrollo económico y científico. El segundo factor se determina a partir de la revolución industrial: el ascenso de una burguesía urbana y la consolidación definitiva del Estado como forma de organización política. En tercer lugar, referenciamos al impulso cultural producto del Renacimiento, en el cual se establecía una mirada donde el hombre y el mundo son uno. El cuarto factor se determinó a partir de la revolución tecnológica, haciendo hincapié en el transporte y la comunicación. Por último, el carácter expansionista producto de la religión cristina, pero quizás lo más significativo fue el sentir superior de la europeidad, la idea de civilización que ha estado presente en todo el proceso de mundialización, justificando su expansión y, por ende, su conquista y posterior colonización.

Como producto de este proceso se fueron concentrando continentes y regiones a partir de la conquista, invasión y colonización europea; se fueron uniendo aquellos lugares que hasta ese entonces permanecían aislados: este proceso se observó en América, Oceanía y gran parte de África; y allí donde existían contactos esporádicos, como con el mundo asiático, las relaciones se volvieron cada vez más estrechas (Truyol, 1993, pp. 27 y ss.).

11 Los diversos factores que proponemos para identificar el proceso histórico de la mundialización han sido trabajados a partir de los siguientes autores: Truyol, 1993; Arenal, 2009; Landes, 1999; Mcneill, 1998; Mokyr, 1993. 
Ya para el siglo XVI, el 90\% de la población se encontraba en una red mundial de intercambios comerciales, políticos, y tecnológicos. Como sostuvo Paul Valéry, "[...] comienza el tiempo del mundo finito" (1954, p. 23).

Desde los efectos que derivaron de la mundialización debemos considerar, en primer lugar, la problemática del espacio y el tiempo en el devenir de la humanidad. Esto no es otra cosa que la integración en una misma sociedad internacional, por lo tanto, el espacio y el tiempo se hacen únicos, mundiales. "Un tiempo y un espacio mundiales que son los propios de Occidente, que se imponen en la nueva sociedad internacional" (Arenal, 2009, p. 200). Un segundo efecto se vislumbra a partir de la creciente interdependencia entre estados, que no solo implica la aparición de nuevos actores, sino la acentuación de la integración de los estados, y muy especialmente, de las ciudades. Como tercer efecto, y consecuencia directa del proceso de mundialización se sostiene que la sociedad internacional es ahora mundial, universal; por lo tanto, es global. Otro de los efectos señalados se determina a partir de una sociedad mundial en gran parte a medida de Occidente, producto de la expansión, dominación y colonización. Por último, la sociedad internacional se caracterizará por su progresiva heterogeneización, en la cual se van a integrar actores con marcadas diferencias y características.

A partir de los procesos y sus efectos se puede definir a la mundialización "[...] como la dinámica que lleva desde un mundo marcado por la existencia de distintas sociedades internacionales particulares, incluso sin contacto entre sí, existente a mediados del siglo XV, hasta un mundo caracterizado por la existencia de una sociedad internacional mundial y universal" (Arenal, 2009, p. 197). De esta menara, se sostiene que la mundialización implica, ante todo, la idea de que el espacio y el territorio se hacen uno solo, siempre considerando que el proceso del espacio y el tiempo fueron una consecuencia propia de Occidente y su imposición en el ámbito global. Coincide Roberto Mesa al afirmar que con la mundialización nos encontramos en "[...] el mundo cerrado de una sociedad internacional herméticamente estatalizada" (1992, p. 113).

En la actualidad el proceso mundialización lo identificamos en el efecto que tiene sobre las ciudades cuando advertimos 
que estas adquieren características globales en el marco de una nueva sociedad internacional:

En definitiva, la dinámica de mundialización no solo ha traído consigo la conformación de una sociedad de dimensiones mundiales, con el consiguiente dominio y unificación del espacio y del tiempo a nivel planetario, sino que además ha dado lugar a una sociedad internacional marcadamente estatocéntrica y heterogénea, sentando algunas de las principales características de la actual sociedad internacional, muchos de sus principales problemas y las bases sobre las que evolucionará hasta el presente (Arenal, 2009, p. 206).

Desde esta perspectiva teórica, la mundialización es un fenómeno que se desarrolla y se conceptualiza antes que el proceso globalizador; sin embargo, no ha sido absorbido por esta. En efecto, la mundialización está vigente a partir de la conjunción entre la dinámica de la globalización, interdependencia y, más recientemente, lo glocal, observado a partir de las diversas estrategias de vinculación que ejercen los actores subnacionales.

\section{La creciente interdependencia}

Como sostuvieron R. Keohane y J. Nye, "[...] vivimos en una era de interdependencia. Esta vaga afirmación expresa pobremente un sobreentendido que, sin embargo, corresponde a un difundido sentimiento de que la propia naturaleza de la política mundial está cambiando" (1988, p. 19). La dinámica de la creciente interdependencia acompaña al proceso de globalización; algunos sostienen que esta es una de las consecuencias lógicas del fenómeno globalizador, pero buscamos desarrollar este efecto para explorar todo su alcance.

El enfoque conocido como interdependencia compleja tuvo su desarrollo a partir de las propuestas trabajadas por Keohane y Nye, dentro del seno del realismo político estadounidense y posteriormente logra alcanzar un análisis más profundo sosteniendo que 
[...] en lenguaje común, dependencia significa un estado en que se es determinado o significativamente afectado por fuerzas externas. Interdependencia, en su definición más simple, significa dependencia mutua. En política mundial, interdependencia se refiere a situaciones caracterizadas por efectos recíprocos entre países o entre actores en diferentes países (1988, p. 22).

La interdependencia incorpora una pluralidad de actores de características no estatales: organismos internacionales, ONG, empresas multinacionales, individuos y, por último, el papel de las ciudades globales en el proceso conocido como glocalización. De esta manera el Estado pierde el rasgo distintivo que pregonaban los tradicionalistas, entrando en competencia con otros actores. Lo local rompe con la lógica clásica de los estados, generando múltiples conexiones.

En igual sentido se ha dicho que "[...] dado que la creciente interdependencia es hoy parte integral de la globalización, sus efectos no pueden separarse de los que se derivan de esta última” (Arenal, 2009, p. 211).

Proponemos destacar tres características esenciales que nos permitirán entender su análisis ${ }^{12}$ :

1. Canales múltiples, canales que conectan de diversas formas a las sociedades. Estos pueden darse de manera formal o informal. Mientras los realistas sostuvieron que solo se producían relaciones entre estados; este enfoque señala que además hay relaciones transgubernamentales, esto es, se producen relaciones transnacionales. De esta manera, las políticas locales cobrar una relevancia que en el juego del pasado dependía exclusivamente de los estados y sus intereses nacionales en el ámbito global.

2. Ausencia de jerarquías: la agenda de los estados estará compuesta por una variedad de temas -más allá del militar- y no hay jerarquía entre ellos: así la seguridad ya no será prioritaria. Este punto se ve a partir de la década

12 Para un análisis mayor sobre las características de la interdependencia compleja véase Keohane y Nye (1988, pp. 41-47). 
de los setenta y la incorporación de una variedad de temas ajenos a los militares. Múltiples temas pueden afectar a un determinado sector o grupo, aunque no afecte a toda la nación. De esta manera se hace más compleja la formulación de una política pública que ahora estará dominada por varios temas.

3. Menor papel de las fuerzas armadas: uno de los preceptos básicos de este enfoque sostiene que en un mundo caracterizado por la interdependencia compleja, los estados no utilizan la fuerza militar contra otros estados. Esto es cierto cuando analizamos la imposibilidad de resolver un conflicto económico entre estados de una misma región. Una de las premisas centrales del realismo era que la fuerza militar subordinaba otros medios de poder; la distensión, la disminución al temor de un ataque por parte de otro Estado, los costos elevados de una invasión o la imposibilidad de solucionar a través de la fuerza problemas tales como la degradación del medioambiente han dejado lugar a la aparición de otros instrumentos de poder más allá del militar.

A partir de este primer análisis surgen tres premisas que dan origen a lo que Keohane y Nye denominan los procesos politicos de la interdependencia compleja ${ }^{13}$ :

1. Estrategias de vinculación: el análisis clásico decía que los estados que tenían el poder militar dominaban el sistema internacional; en un mundo caracterizado por la interdependencia, la fuerza militar se encuentra desvalorizada. De esta manera, los estados fuertes se vinculan a través de la fuerza y los más débiles se enlazan generalmente en los organismos internacionales. La disminución del poder militar lleva a que los estados generen otras estrategias de vinculación. En las últimas décadas lo local ha comenzado a ser el centro de las

13 Para un análisis mayor sobre las características de los procesos políticos de la interdependencia compleja véase Keohane y Nye 1988, pp. 47-56. 
estrategias de vinculación entre los estados, ciudades globales que piensan globalmente y actúan localmente.

2. Establecimiento de la agenda: la falta de una jerarquía de temas determina relaciones más complejas; las cuestiones militares seguirán en la agenda, pero en un mundo de interdependencia compleja ciertos temas adquirirán una relevancia preponderante: la política monetaria, cuestiones de medioambiente, los flujos de personas, la influencia de las empresas multinacionales, la articulación de demandas de la sociedad civil y el papel de las ciudades globales se constituyen en temáticas importantes en la agenda.

3. Relaciones transnacionales y transgubernamentales: las dos características señaladas anteriormente hacen cada vez más difícil una distinción clara entre lo doméstico y lo internacional. La multiplicidad de actores y socios hace más compleja esta relación: se establecen relaciones entre los estados, las ciudades y los actores no estatales.

La interdependencia afecta la política mundial y el comportamiento de los estados, pero las acciones gubernamentales también influyen sobre los modelos de interdependencia: al aceptar procedimientos, normas o instituciones para ciertas clases de actividades, los gobiernos regulan y controlan las relaciones transnacionales e interestatales.

La interdependencia compleja nos permite establecer patrones de conducta diferentes a la concepción realista; si bien es conveniente destacar que no se alejan por completo de sus desarrollos, podemos señalarla como el primer cuestionamiento serio que se les hacen a los teóricos clásicos.

En un mundo complejo este enfoque nos permite realizar un mejor aproximamiento de nuestro estudio, porque comprendemos la interdependencia, no solo entre, estados sino entre estos, las sociedades civiles, las organizaciones no gubernamentales, las empresas y las ciudades ${ }^{14}$. Como sostuvo Nye, los procesos

14 En ese sentido Arenal señala: "El constante incremento de los niveles de interdependencia ha transformado radicalmente la sociedad internacional y de manera muy especial el comportamiento de los estados, contribuyendo de manera decisiva a conformar una sociedad internacional postwestfaliana, como consecuencia de los decisivos efectos que se han derivado de esta dinámica” (2009, p. 209). 
de transformación que genera la interdependencia compleja en la sociedad internacional, tanto en lo económico, político, sociocultural y científico-tecnológico "[...] elevaron la importancia de los asuntos transnacionales y facultaron a diferentes actores para tener un papel más amplio en la política mundial” (2003, p. 2). Por esto es que identificamos la participación ineludible de una variedad de actores además de los estados nacionales. Un escenario que se visualiza como favorable para la participación de actores subnacionales, pese a que no son considerados como sujetos del derecho internacional, aunque intervienen en diversas modalidades de acuerdos, entendimientos y hermanamientos de cooperación internacional de manera formal o informal, y desde ámbitos económicos o culturales.

\section{Dialéctica global-local: la política internacional subnacional}

El escenario internacional al que denominamos sociedad internacional global presenta una nueva dialéctica sobre la cuestión global-local. Por lo tanto, debemos considerar las "transformaciones globales"15 sobre este contexto, en un proceso que implica, según Beck, "[...] un cambio de paradigma científico y un cambio de paradigma político" (2008, p. 129).

La visión realista de las relaciones internacionales y la hipótesis del Estado como único actor del sistema internacional ya no son un modelo teórico válido. La globalización supone dos sucesos. En primer lugar, las reglas y, por sobre todo, los conceptos centrales de esa visión teórica pierden su función explicativa: conceptos como "Estado nacional”, "capitalismo nacional”, "soberanía nacional”, entre otros, pierden su utilidad porque refieren situaciones y valores que ya no describen la compleja realidad del sistema internacional y, por ende, no pueden explicarlo.

En segundo lugar, el proceso globalizador permite generar nuevos espacios y, por lo tanto, genera nuevos conceptos adaptados a una nueva realidad. De esta manera, "[...] la política se deslimita y

15 El concepto de transformaciones globales es trabajado en extenso por los académicos Held et al. (2002). 
desestataliza” (Beck, 2008, p. 27). En este proceso aparecen nuevos actores, con nuevos papeles, con reglas diferentes, generando la necesidad de identificar conceptos que se adaptan a una sociedad en cambio.

Entre esos nuevos conceptos aparece el de glocaly preguntas asociadas: ¿lo local tiene una entidad que no depende lo global?, ¿lo local se determina, por contraposición, a lo global?, ¿lo local se constituye/reafirma a partir de una reacción a la globalización?, ¿O es, por el contrario, una aceptación pasiva de los impactos del proceso globalizador?

Ante las limitaciones estatales, se ha hecho cada vez más notoria la posibilidad de una relación entre el desarrollo local y las dinámicas propuestas desde el ámbito global. El gobierno local implica el instrumento que impulsa la actividad internacional:

Por un lado, en clave económica, para la creación de lazos internacionales tendientes a estimular varias cuestiones que potencien y refuercen el desarrollo local, entre otras, el comercio, la inversión y el turismo. Por otro lado, en clave política, el municipio es una herramienta fundamental para sostener la relación entre el desarrollo local y la dimensión externa, a través del diseño de un esquema de influencia institucional sobre las estructuras del gobierno central (Miranda, 2005, p. 7).

"Pensar globalmente, actuar localmente", señala una apertura de lo particular a lo universal y este es justamente el desafío que se presenta a los actores subnacionales en la actual sociedad internacional. Entendemos a los municipios como los actores centrales, y, como sostuvo Di Pietro, “[...] lo local es la entidad subnacional de análisis, planificación y acción para el desarrollo que implica una serie de relaciones, comportamientos, pautas y convenciones comunes" (2001, p. 23).

Podemos continuar conceptualizando el término local, ya que hay una variedad de definiciones, pero en nuestro caso preferimos optar por una caracterización. Lo local es territorial, porque se despliega en un espacio determinado (municipal o regional); multidimensional, ya que abarca las distintas facetas de la vida humana; sistémico, porque supone la cooperación 
de los diversos actores de una comunidad; integrado, ya que allí se articulan políticas públicas locales (presupuestos participativos); institucionalizado, se establecen reglas de juegos que regulan las conductas locales, e innovador, porque fomenta la participación social.

Castells señaló que "[1]a identidad territorial está en las raíces del alza mundial de gobiernos locales y regionales como actores significativos de representación e intervención, más apropiados para adaptarse a la variación interminable de los flujos globales" (1998, p. 394). Como ha sostenido en nuestro ámbito Parmigiani de Barbará, las lógicas de lo global y lo local se encuentran unidas en la dialéctica de la realidad de nuestro tiempo, complementándose, y no excluyéndose. Agrega:

Es un escenario caracterizado por una intensa globalidad que tiende a uniformar prácticas sociales y culturales así como diagnósticos y soluciones por parte de los decisores políticos y económicos, tanto al interior cuanto hacia el exterior del Estado-nación; simultáneamente, se visualiza con claridad otro escenario, superponiéndose al anterior, y que refleja la afirmación de peculiaridades organizativas, económicas y socioculturales desde las unidades políticas diversas (Parmigiani de Barbará, 2002, p. 1).

Esta influencia e interdependencia de lo global en lo local y este auge de los gobiernos locales es lo que resume el neologismo "glocal". Esto debe ser considerado a partir de la dinámica que se impone entre la globalización, la interdependencia compleja y la mundialización, entendiendo que dicho concepto se determina como un proceso de la caducidad relativa del paradigma realista. Siguiendo a Marramao (2006, p. 54), sostenemos que estamos en un vacío político que se caracterizará por el paso entre el no más del viejo orden estatal al todavía no de un nuevo orden internacional. Pretendemos señalar que en este escenario se ubica lo glocal, quizás en una suerte de tenaza entre unilateralidades que se encierran unas a otras.

¿Dónde se observa la dialéctica existente entre lo global y lo local? La propuesta entiende a la política internacional subnacional como una política pública que permite a los gobiernos locales actuar 
como actores globales. En la actualidad esto se observa en múltiples actividades que desarrollan estos sujetos: acuerdos bilaterales, hermanamientos de ciudades, proyectos de cooperación técnica y financiera, realización de intercambios culturales, deportivos, turísticos y de buenas prácticas en la gestión pública, participación y organización de ferias y congresos internacionales, entre otras actividades.

Dentro de las herramientas y el accionar disponibles que presenta la política internacional subnacional pretendemos referenciar brevemente el abanico disponible siempre teniendo en cuenta que “[...] la participación externa de las entidades subnacionales es una política pública local, que materializa y aplica la estrategia de un gobierno de este nivel en el campo de las relaciones internacionales" (Calvento, 2016, p. 309).

Mariana Calvento (2016) realiza una conceptualización, que seguiremos en nuestro desarrollo operativizando y sistematizando: la política internacional subnacional tiene dos dimensiones, de relacionamiento institucional internacional y de posicionamiento internacional.

En relación con las herramientas de relacionamiento, se destacan los acuerdos bilaterales ${ }^{16}$, donde cobran un papel protagónico los hermanamientos de ciudades y, en menor medida, acuerdos y convenios de cooperación internacional bilateral. Dentro de estas herramientas surgen las relaciones multilaterales, en las cuales se destaca la creación de redes de ciudades que permiten una mayor generación de políticas de promoción e imagen de las ciudades. En nuestra región la red principal de municipios es la Red de Mercociudades, la cual es la mayor red regional de participación de estrategias de relacionamiento institucional regional.

En cuanto al posicionamiento internacional, los municipios buscan generar acuerdos de carácter multilateral con el objetivo de lograr posicionamiento exterior. En una variedad de herramientas destacamos el desarrollo de políticas locales de comercio exterior cuyo objetivo prioritario se establece en generar condiciones favorables para el desarrollo de economías locales y regionales.

16 Los acuerdos bilaterales "[...] representan instrumentos concretos, tangibles y factibles de cooperación, asentados en una base común de voluntades e intereses a corto, mediano y largo plazo" (Batista, Jakobsen y Evangelista, 2008, p. 59). 
Otras de las estrategias es la conocida como marketing internacional, la cual busca señalar las ventajas comparativas de una localidad; en este sentido, se destaca la tan promocionada marca-ciudad, en la cual se destacan los que identifican a una ciudad.

De manera breve, hemos identificado el auge en la participación internacional de los actores subnacionales, identificando sus herramientas y estrategias, desde diversas dimensiones; esto permite observar la búsqueda de escenarios de cooperación, intercambio y posicionamiento en el ámbito internacional. A partir de estas consideraciones podemos ahora entender la globalización como el ámbito ideológico y a los territorios locales como los ámbitos de aplicación de esa ideología en un proceso de interdependencia compleja. Como ya se ha señalado, "[1]o global (lo grande) solo puede ser comprendido en función de lo concreto, lo pequeño, lo local” (Beck, 2008, pp. 76-77).

\section{Consideraciones finales}

En este ensayo hemos discutido conceptos clave de las relaciones internacionales, cuestionando ciertos paradigmas y modelos de comprensión, como los del realismo político, que han sido claves y fundamentales para esta disciplina.

Hemos sostenido que las dinámicas políticas locales son influenciables por los contextos globales, siendo el Estado nacional incapaz de moldear modificaciones/adaptaciones a los paradigmas internacionales vigentes. Estos se imponen sobre la lógica del Estado-nación. Por lo tanto, buscamos la deconstrucción y reconstrucción de aportes teóricos generados desde las relaciones internacionales intentando identificar las características del proceso globalizador, la creciente interdependencia, la mundialización y la dialéctica entre lo global-local.

Los mecanismos de cambios generados hacia fines del siglo XX han tenido varias aristas: la profundidad de las cuestiones económicas internacionales a partir de 1970, sobre todo en el desarrollo del comercio y las finanzas internacionales; el desarrollo en materia de seguridad internacional a partir de la amplificación de las agendas; la apertura de la diplomacia, y la creciente 
gravitación internacional de nuevos actores no tradicionales. En el marco de una sociedad internacional global se generaron nuevos escenarios y, por lo tanto, nuevas políticas públicas.

A lo largo del trabajo pudimos moldear un estado del arte desde el cual abordamos a la globalización como un fenómeno contemporáneo y determinado a partir de su multidimensionalidad, distinguiendo los desafíos que se plantea para los estados; en particular, cómo este contexto repercute en la formulación de políticas internacionales subnacionales.

¿Qué sucede entre la mundialización y la globalización? La respuesta, según nuestra formulación, es que la mundialización antecede a la globalización, pues pretende generar una sociedad internacional distinta a la que pretende el proceso globalizador y destacamos que estas dinámicas encuentran sus centros, para reforzarse y generar mecanismos de cambios en el contexto de la sociedad internacional global. En efecto, el proceso globalizador se entrelaza con otros procesos: la mundialización lo antecede, la interdependencia lo acrecienta y la dialéctica global-local genera oportunidades y desafíos.

Desde nuestra discusión teórica y metodológica, la interacción entre los tres procesos señalados y su vinculación en la dialéctica global-local se propuso referenciar la creciente participación de actores subnacionales (municipales o regionales) a partir de diversas estrategias y herramientas que abarcan diversas formas de relacionamiento y posicionamiento internacional. Pretendemos destacar el papel activo y el interés de estos actores que van desarrollando a partir de la generación de espacios de cooperación e intercambio. Creemos que su relevancia viene dada, en primer lugar, por el desarrollo propuesto en este trabajo, el cual genera un debate y cuestiona las relaciones internacionales y al enfoque realista dominante en nuestra disciplina, y, en segundo lugar, por establecer una dialéctica que nos permite determinar los rasgos que ha adquirido la política internacional subnacional y sus herramientas disponibles.

En definitiva, lo local y lo global no se excluyen, sino que, por el contrario, lo local debe ser pensado como parte integral de lo global en una necesaria reconceptualización que nos impone la nueva sociedad internacional global. 


\section{Referencias}

Arenal, C. (2005). En torno al concepto de sociedad internacional. En soberanía del Estado y derecho internacional: homenaje al profesor Juan Antonio Carrillo (pp. 453-464). Sevilla: Universidad de Córdoba/ Universidad de Sevilla/Universidad de Málaga.

Arenal, C. (2009). Mundialización, creciente interdependencia y globalización en las relaciones internacionales. En V. Gasteiz (ed), Cursos de derecho internacional y relaciones internacionales (pp. 181-268). Bilbao, Universidad del País Vasco.

Batista, S., Jakobsen, K. y Evangelista, A. (2008). La apertura al exterior de las ciudades latinoamericanas y la cooperación descentralizada. Montevideo: Observatorio de Cooperación Descentralizada Unión Europea América Latina.

Bauman, Z. (2010) La globalización: consecuencias humanas (4a. ed.) Buenos Aires: Fondo de Cultura Económica.

Beck, U. (2008) ¿Qué es la globalización? Falacias del globalismo, respuestas a la globalización. Buenos Aires: Paidós.

Bull, H. (1977). The Anarchical Society. New York: Columbia University Press.

Buzan, B. y Little, R. (2000). International Systems in World History. Remaking the Study of International Relations. Oxford: Oxford University Press.

Calduch Cervera, R. (2000). Métodos y Técnicas de Investigación en relaciones internacionales. Madrid: Universidad Complutense de Madrid.

Calvento, M. (2016). La política internacional subnacional: una propuesta para el abordaje del accionar contemporáneo en Argentina. Desafios, 28(I), 295-332, doi: dx.doi.org/10.12804/desafios28.1.2016.07

Castells, M. (1998) La era de la información (vol. 2). Madrid: Alianza Editorial.

Comisión Económica para América Latina y el Caribe [Cepal]. (2002). Globalización y desarrollo. Vigésimo periodo de sesiones. Brasilia, Brasil. Recuperado de http://www.eclac.org

Costamagna, P. y Foglia, M. (2011). Hacia la construcción de una agenda estratégica de internacionalización de los territorios. Documento de trabajo del Programa de Competitividad Territorial Región Central de la provincia de Santa Fe. Rafaela: BID-OMIN.

Deutsch, K. (1966). The Nerves of Government: Models of Political Communication and Control. New York: The Free Press.

Duroselle, J. B. (1998). Todo imperio perecerá. México: Fondo de Cultura Económica.

Giddens, A. (1993) Consecuencias de la modernidad. Madrid: Alianza.

Held, D. (1997). Democracy and Globalization. Working Paper 97/5. MaxPlanck-Institute for the Study of Societies.

Held, D. y McGrew, A. (2003). Globalización/antiglobalización. Sobre la reconstrucción del orden mundial. Barcelona: Paidós. 
Held, D. (2014). ¿Hay que regular la globalización? La reinvención de la política. En J. Corbetta, J. Marchionni y R. Piana (eds.), Nuevos ensayos sobre la democracia contemporánea (pp.,50-68) $1^{\mathrm{a}}$ ed). Buenos Aires: el autor.

Held, D., McGrew, A., Goldblatt, D. y Perraton, A. (2002). Transformaciones globales. Política, economía y cultura, México: Oxford University Press.

Hoffmann, S. (1963) Teorías contemporáneas de las relaciones internacionales. Madrid: Tecnos.

Keohane, R. y Nye, J. (1988). Poder e interdependencia. Buenos Aires: GEL.

Landes, D. S. (1999). La riqueza y la pobreza de las naciones. Por qué algunas son tan ricas y otras tan pobre. Barcelona: Crítica.

Maira, L. (Ed.) (2010). La política internacional subnacional en América Latina. Buenos Aires: Libros del Zorzal.

Márcio Cruz, P. y Bodnar, Z. (2008). Pensar globalmente y actuar localmente: el estado transnacional ambiental en Ulrich Beck. Jurididicas, 5 (2), 13-25.

Marramao, G. (2006) Pasaje a Occidente. Buenos Aires: Katz.

Merle, M. (1986) Sociología de las relaciones internacionales. Madrid: Alianza.

Miranda, R. (2005). Paradiplomacia y gobierno local: indicios de un modo diferente de hacer Relaciones Internacionales. Instituto de Relaciones Internacionales.

Parmigiani de Barbará, M. C. (2002) La coordinación intergubernamental subnacional de cara a la crisis: posibilidades y límites. VII Congreso del CLAD.

Ribas Mateos, N. (2002). El debate sobre la globalización. Barcelona: Ediciones Bellaterra.

Robertson, R. (1992). Globalization: Social Theory and Global Culture. Londres: Sage.

Touraine, A. "La globalización como ideología”, diario El País, el 29 de septiembre de 1996. Recuperado de https://elpais.com/ diario/1996/09/29/opinion/843948007_850215.html (18/09/2017).

Truyol, A. (1993). La sociedad internacional. Madrid: Alianza.

Valéry, P. (1954). Miradas al mundo actual. Buenos Aires: Losada.

Waltz, K. (1979). Teoría de la Política Internacional. Buenos Aires: GEL. 